\title{
Stealth Communication with Vanishing Power over Binary Symmetric Channels
}

\author{
Diego Lentner and Gerhard Kramer \\ Institute for Communications Engineering, Technical University of Munich, 80290 Munich, Germany \\ Email: \{diego.lentner, gerhard.kramer\}@tum.de
}

\begin{abstract}
A framework for stealth communication with vanishing power (VP) is presented by studying binary symmetric channels. Coding theorems are proved by modifying Gallager's error exponents for VP and by applying resolvability exponents. The analysis unifies and generalizes existing rate bounds for covert and stealth communication.
\end{abstract}

\section{INTRODUCTION}

Covert communication [1] refers to a scenario where a sender Alice communicates with a receiver Bob without a third party, Warren, being able to detect the communication. In contrast to the secrecy problem, it is not the content of the message that Alice and Bob want to hide from Warren but the presence of the message itself. Bash et al. [1] showed that on the order of $\sqrt{n}$ bits can be covertly communicated in $n$ channel uses over additive white Gaussian noise (AWGN) channels. This square root law also applies to discrete memoryless channels (DMCs) [2], [3].

Covertness can be measured by the informational divergence of two types of channel output statistics: those when a meaningful message is transmitted and those when a sequence of "zero" symbols is transmitted, where the "zero" symbol usually represents the absence of energy. Second-order asymptotics for various covertness measures are derived in [4]. Covert communication may require shared randomness between Alice and Bob in the form of a secret key, unless Warren's channel from Alice is noisier than Bob's, as shown in [5], [6] for binary symmetric channels (BSCs).

Stealth communication generalizes covert communication by discarding the requirement that Alice must be silent when not communicating information to Bob, i.e., Alice is free to transmit symbols other than the "zero" symbol. The idea is that Alice confuses Warren by sending obfuscating symbols. Obfuscation is an old technique to enhance privacy, e.g., it can hide personal information such as mobility patterns or web browsing behavior.

One can show formally [7], [8] that obfuscation can break the square root law, and in fact communication with positive rate is possible without Warren being able to detect meaningful communication. The price that Alice pays is that she must consume more energy than for covert communication, and we thus arrive at a capacity-cost tradeoff. This tradeoff depends on which obfuscation patterns are permitted, and we will consider obfuscation strings consisting of independent and identically distributed (i.i.d.) channel symbols.

The main contribution of this work is two-fold.
1) We introduce a framework for stealth communication that includes previously treated scenarios as special cases. In particular, we are interested in using vanishing power, as for covert communication, but with energy that scales as $n^{\alpha}, 0 \leq \alpha<1$, with blocklength $n$. Observe that covert communication has $\alpha \leq 1 / 2$ while stealth communication as treated in [7], [8] has $\alpha=1$.

2) We prove coding theorems by using suitably modified Gallager exponents. This gives an alternative, and we believe simpler, approach to prove and understand achievability as compared to previous work.

This paper is organized as follows. Sec. [II introduces notation and classic error exponents. In Sec. III] we derive achievable codebook scaling constants for vanishing power (VP) communication by using modified error exponents. We apply these results in Sec. IV] to prove achievability of VP stealth communication. Finally, we compare our results for the covert communication case with bounds from [2], [3], [6] in Sec. $\nabla$.

\section{Preliminaries}

\section{A. Notation}

Random variables are denoted by upper case letters and their realizations by the corresponding lower case letters. Finite sequences of random variables are written with a superscript indicating the number of symbols of the sequence, e.g., $X^{n}=X_{1}, \ldots, X_{n}$. Let $X$ be a discrete random variable with probability distribution $P_{X}$ and alphabet $\mathcal{X}$. If the symbols $X_{i}$, $i=1, \ldots, n$, are i.i.d. according to $P_{X}$, then the distribution of $X^{n}$ is $P_{X^{n}}\left(x^{n}\right)=\prod_{i=1}^{n} P_{X}\left(x_{i}\right) \triangleq P_{X}^{n}\left(x^{n}\right)$. For any two probability distributions $P_{X}$ and $P_{\tilde{X}}$ on $\mathcal{X}$ where $P_{X} \ll P_{\tilde{X}}$, i.e. $P_{\tilde{X}}(x)=0 \Rightarrow P_{X}(x)=0$ for any $x \in \mathcal{X}$, the informational divergence between $P_{X}$ and $P_{\tilde{X}}$ is

$$
\mathbb{D}\left(P_{X} \| P_{\tilde{X}}\right) \triangleq \sum_{x: P_{X}(x)>0} P_{X}(x) \log \frac{P_{X}(x)}{P_{\tilde{X}}(x)}
$$

and their variational distance is defined as

$$
\mathbb{V}\left(P_{X} \| P_{\tilde{X}}\right) \triangleq \frac{1}{2} \sum_{x \in \mathcal{X}}\left|P_{X}(x)-P_{\tilde{X}}(x)\right| .
$$

Informational divergence and variational distance are related by Pinsker's inequality:

$$
\mathbb{V}\left(P_{X} \| P_{\tilde{X}}\right)^{2} \leq \frac{1}{2} \mathbb{D}\left(P_{X} \| P_{\tilde{X}}\right) .
$$


The chi-squared distance of $P_{X}$ and $P_{\tilde{X}}$ is

$$
\chi_{2}\left(P_{X} \| P_{\tilde{X}}\right) \triangleq \sum_{x \in \mathcal{X}} \frac{\left(P_{X}(x)-P_{\tilde{X}}(x)\right)^{2}}{P_{\tilde{X}}(x)} .
$$

The mutual information of $X$ and $Y$ is denoted $I\left(P_{X} ; P_{Y \mid X}\right)$.

\section{B. Error Exponents}

Let $W$ be Alice's message and let $\hat{W}$ be Bob's estimate of this message. Gallager used a random coding argument to show that if each message $W$ selects a codeword from a code of cardinality $M$ and length $n$, then the worst-case error probability under maximum-likelihood (ML) decoding over a noisy channel $P_{Y^{n} \mid X^{n}}$ can be bounded as [9, Ch. 5]

$$
\begin{aligned}
& \operatorname{Pr}[\hat{W} \neq 1 \mid W=1] \\
& \leq(M-1)^{\rho} \sum_{y^{n}}\left\{\sum_{x^{n}} P_{X^{n}}\left(x^{n}\right) P_{Y^{n} \mid X^{n}}\left(y^{n} \mid x^{n}\right)^{\frac{1}{1+\rho}}\right\}^{1+\rho}
\end{aligned}
$$

where $\rho, 0 \leq \rho \leq 1$, is an optimization parameter.

Consider a discrete memoryless source (DMS) with probability distribution $P_{X}$ and a DMC $P_{Y \mid X}$. Define the code rate as $R=\frac{\log M}{n}$. We have $(M-1)^{\rho} \leq M^{\rho}=e^{n R \rho}$ and (5) becomes (see [9])

$$
\operatorname{Pr}[\hat{W} \neq 1 \mid W=1] \leq e^{-n E_{G}\left(R, P_{X}\right)}
$$

where the error exponent $E_{G}\left(R, P_{X}\right)$ is defined as

$$
\begin{aligned}
& E_{G}\left(R, P_{X}\right)=\max _{0 \leq \rho \leq 1}\left[E_{0}\left(\rho, P_{X}\right)-\rho R\right] \\
& E_{0}\left(\rho, P_{X}\right)=-\log \sum_{y}\left\{\sum_{x} P_{X}(x) P_{Y \mid X}(y \mid x)^{\frac{1}{1+\rho}}\right\}^{1+\rho} .
\end{aligned}
$$

\section{ERROR EXPONENTS FOR VP COMMUNICATION}

This section considers classic point-to-point communication over a $\mathrm{BSC}$ and with VP. Let $\operatorname{BSC}(p)$ denote a BSC with cross-over probability $p$. We define the energy of the binary sequence $X^{n}$ as its Hamming weight $\sum_{i=1}^{n} X_{i}^{2}$. In the following, the code length $n$ is a free parameter and we transmit only one codeword (one-shot analysis).

\section{A. Information Rate Analysis}

Suppose we have the average block power constraint

$$
\frac{1}{n} \mathbb{E}\left[\sum_{i=1}^{n} X_{i}^{2}\right] \leq \frac{a n^{\alpha}}{n}
$$

where $0 \leq \alpha \leq 1$ and $0<a$. The constraint can be satisfied by choosing the channel input distribution as $P_{X, n}(1)=1-P_{X, n}(0)=\frac{a n^{\alpha}}{n}$. Note that the distribution $P_{X, n}$ directly depends on the choice of the blocklength $n$, which we emphasize with the additional subscript. Clearly, if $0 \leq \alpha<1$ then the power of $X^{n}$ will vanish for $n \rightarrow \infty$. We therefore refer to signaling with $0 \leq \alpha<1$ as VP transmission.
We assess how much information can be transmitted with VP over a $\operatorname{BSC}(p)$. Let the transmitted signal $X^{n}$ be distributed according to $P_{X, n}$. The receiver observes the binary sequence $Y^{n}$ which is distributed as

$$
\begin{aligned}
P_{Y, n}(0) & =\left(1-\frac{a n^{\alpha}}{n}\right) \bar{p}+\frac{a n^{\alpha}}{n} p \\
& =\bar{p}-(1-2 p) \cdot \frac{a n^{\alpha}}{n} \\
P_{Y, n}(1) & =\left(1-\frac{a n^{\alpha}}{n}\right) p+\frac{a n^{\alpha}}{n} \bar{p} \\
& =p+(1-2 p) \cdot \frac{a n^{\alpha}}{n}
\end{aligned}
$$

where we introduced the shorthand $\bar{p}=1-p$. The mutual information is

$$
\begin{aligned}
& I\left(P_{X, n} ; P_{Y \mid X}\right)=H\left(P_{Y, n}\right)-H_{2}(p) \\
& \quad=H_{2}\left(p+(1-2 p) \cdot \frac{a n^{\alpha}}{n}\right)-H_{2}(p) \\
& \quad \approx(1-2 p) \frac{a n^{\alpha}}{n} \cdot \log \frac{\bar{p}}{p}
\end{aligned}
$$

where $H_{2}(p)=-p \log p-(1-p) \log (1-p)$ and where we have used the first-order Taylor expansion

$$
\left.H_{2}(x)\right|_{x=p} \approx H_{2}(p)+\left.(x-p) \cdot \frac{\partial H_{2}(x)}{\partial x}\right|_{x=p} .
$$

\section{B. Modified Random Coding Exponent}

Directly applying the error exponent framework introduced in Sec. II-B to our model has $E_{0}\left(\rho, P_{X, n}\right)$ scaling with $n^{\alpha} / n$ which goes to zero as $n$ increases. To get a more meaningful exponent, we normalize (8) by the scaling factor $n^{\alpha} / n$ and compute

$$
\hat{E}_{0}^{\alpha}\left(\rho, P_{X, n}\right)=\lim _{n \rightarrow \infty} \frac{n}{n^{\alpha}} E_{0}\left(\rho, P_{X, n}\right) .
$$

Now define $R_{\alpha}=\frac{1}{n^{\alpha}} \log M$ and a modified error exponent

$$
\hat{E}_{G}^{\alpha}\left(R_{\alpha}, P_{X, n}\right)=\max _{0 \leq \rho \leq 1}\left(\hat{E}_{0}^{\alpha}\left(\rho, P_{X, n}\right)-\rho R_{\alpha}\right)
$$

to describe the error probability decay with $n^{\alpha}$ as

$$
\operatorname{Pr}[\hat{W} \neq 1 \mid W=1] \leq e^{-n^{\alpha} \hat{E}_{G}^{\alpha}\left(R_{\alpha}, P_{X, n}\right)}
$$

for large $n$.

In the following we show that the modified error exponents exhibit similar properties as the well-studied Gallager exponents reviewed in Sec. II-B For the BSC channel model with input $P_{X, n}$ as defined in Sec. III-A, the expression (14) can be explicitly derived:

$$
\begin{aligned}
\hat{E}_{0}^{\alpha}\left(\rho, P_{X, n}\right)=\lim _{n \rightarrow \infty} \frac{n}{n^{\alpha}}\left[-\log \left(\left(\left(1-\frac{a n^{\alpha}}{n}\right) \bar{p}^{\frac{1}{1+\rho}}+\frac{a n^{\alpha}}{n} p^{\frac{1}{1+\rho}}\right)^{1+\rho}\right.\right. \\
\left.\left.+\left(\left(1-\frac{a n^{\alpha}}{n}\right) p^{\frac{1}{1+\rho}}+\frac{a n^{\alpha}}{n} \bar{p}^{\frac{1}{1+\rho}}\right)^{1+\rho}\right)\right] \\
= \begin{cases}(1+\rho) a\left(\bar{p}^{\frac{1}{1+\rho}}-p^{\frac{1}{1+\rho}}\right)\left(\bar{p}^{\frac{\rho}{1+\rho}}-p^{\frac{\rho}{1+\rho}}\right), & \alpha<1 \\
E_{0}\left(\rho, P_{X, n}\right), & \alpha=1 .\end{cases}
\end{aligned}
$$


The complete derivation of (18) involves L'Hospital's rule and is omitted due to space limitations. For the extremal values of $\rho$, we have for $0 \leq \alpha<1$ :

$$
\begin{aligned}
& \lim _{\rho \rightarrow 1} \hat{E}_{0}^{\alpha}\left(\rho, P_{X, n}\right)=2 a(\sqrt{\bar{p}}-\sqrt{p})^{2} \\
& \lim _{\rho \rightarrow 0} \hat{E}_{0}^{\alpha}\left(\rho, P_{X, n}\right)=0 .
\end{aligned}
$$

Finally, the maximum scaling constant $R_{\alpha}$ for which the modified error exponent (15) is positive, and therefore the error probability vanishes for large $n$, is given by

$$
\begin{aligned}
R_{\alpha, \max }\left(P_{X, n}\right)=\left.\frac{\partial \hat{E}_{0}^{\alpha}\left(\rho, P_{X, n}\right)}{\partial \rho}\right|_{\rho=0} \\
=a\left\{\left(\bar{p}^{\frac{1}{1+\rho}}-p^{\frac{1}{1+\rho}}\right)\left(\bar{p}^{\frac{\rho}{1+\rho}}-p^{\frac{\rho}{1+\rho}}\right)+\frac{1}{1+\rho} \cdot\right. \\
\quad\left[\left(\bar{p}^{\frac{1}{1+\rho}}-p^{\frac{1}{1+\rho}}\right)\left(\bar{p}^{\frac{\rho}{1+\rho}} \cdot \log \bar{p}-p^{\frac{\rho}{1+\rho}} \cdot \log p\right)\right. \\
\left.\left.\quad-\left(\bar{p}^{\frac{1}{1+\rho}} \cdot \log \bar{p}-p^{\frac{1}{1+\rho}} \cdot \log p\right)\left(\bar{p}^{\frac{\rho}{1+\rho}}-p^{\frac{\rho}{1+\rho}}\right)\right]\right\}_{\rho=0} \\
=a(1-2 p) \log \frac{\bar{p}}{p} .
\end{aligned}
$$

Observe that the right-hand side (RHS) of (21) is the same as the RHS of (12) after normalizing by $n^{\alpha} / n$. The error probability thus decays exponentially with $n^{\alpha}$ if

$$
n^{\alpha} R_{\alpha} \lesssim n I\left(P_{X, n} ; P_{Y \mid X}\right)
$$

which for $\alpha=1$ reduces to $R<I\left(P_{X} ; P_{Y \mid X}\right)$.

\section{Stealth Communication with VP Obfuscation}

Consider now the stealth communication problem depicted in Fig. 1 Alice wants to transmit a message reliably to Bob over the memoryless channel $P_{Y \mid X}$ without being detected by Warren. Warren observes the output of the channel $P_{Z \mid X}$ and makes a binary hypothesis test whether Alice has transmitted information or obfuscation symbols. Suppose the channel $P_{Y \mid X}$ from Alice to $\operatorname{Bob}$ is a $\operatorname{BSC}(p)$, and the channel $P_{Z \mid X}$ from Alice to Warren is a $\operatorname{BSC}(q)$. We do not restrict the values of $p$ and $q$ other than $p \leq 1 / 2$ and $q \leq 1 / 2$. Suppose that Alice sends with VP as in (9) when transmitting information, and that she sends i.i.d. sequences $X^{n}$ with VP given by

$$
\frac{1}{n} \mathbb{E}\left[\sum_{i=1}^{n} X_{i}^{2}\right]=\frac{b n^{\beta}}{n}, \quad 0 \leq \beta<1,0<b .
$$

when transmitting obfuscation symbols. In the following, we derive conditions on $a, \alpha, b, \beta$ to achieve stealth communication for both uncoded and coded transmission.

\section{A. Uncoded Stealth}

Let $P_{X, n}$ and $P_{X_{o}, n}$ denote the marginals of input distributions satisfying (9) and (23), respectively. Let $P_{Z, n}$ and $P_{Z_{o}, n}$ be the corresponding marginals of the distributions $P_{Z^{n}}$ and $P_{Z_{o}}^{n}$, respectively, which Warren observes at the output of his $\operatorname{BSC}(q)$ from Alice. To prevent Warren from detecting the communication with Bob, Alice must ensure that

$$
\mathbb{D}\left(P_{Z^{n}} \| P_{Z_{o}}^{n}\right) \leq \delta
$$

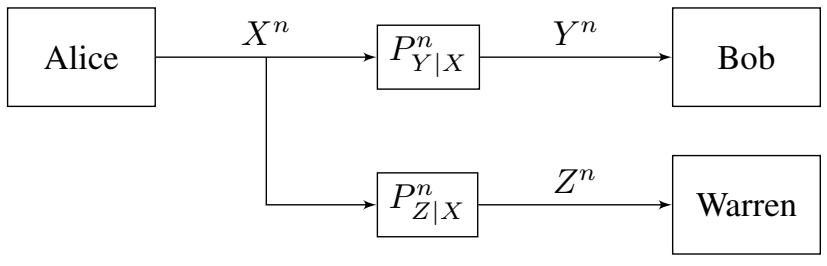

Fig. 1. Stealth communication problem.

for a small constant $\delta>0$.

Following [2], we first consider an uncoded stealth scenario, where $Z^{n}$ is i.i.d., i.e., $P_{Z^{n}}=P_{Z}^{n}$. The stealth constraint (24) is then

$$
n \mathbb{D}\left(P_{Z, n} \| P_{Z_{o}, n}\right) \leq \delta .
$$

Let $\boldsymbol{\mu}_{n}=\left[\begin{array}{ll}\frac{a n^{\alpha}}{n} & \frac{b n^{\beta}}{n}\end{array}\right]^{T}$. We write $\mathbb{D}\left(P_{Z, n} \| P_{Z_{o}, n}\right)$ as a function of $\boldsymbol{\mu}_{n}$ and use

$$
\mathbb{D}\left(P_{Z, n} \| P_{Z_{o}, n}\right)=\frac{1}{2} \frac{(\bar{q}-q)^{2}}{q \bar{q}}\left(\frac{a n^{\alpha}}{n}-\frac{b n^{\beta}}{n}\right)^{2}+o\left(\left\|\boldsymbol{\mu}_{n}\right\|^{2}\right) .
$$

To prove (26), note that the second-order Taylor approximation for a scalar function $g: \mathcal{X}^{n} \rightarrow \mathbf{R}$ around a point $\boldsymbol{x}_{0}$ is

$$
\begin{aligned}
g(\boldsymbol{x})= & g\left(\boldsymbol{x}_{0}\right)+\left.\nabla g(\boldsymbol{x})^{T}\right|_{\boldsymbol{x}=\boldsymbol{x}_{0}}\left(\boldsymbol{x}-\boldsymbol{x}_{0}\right) \\
& +\left.\frac{1}{2}\left(\boldsymbol{x}-\boldsymbol{x}_{0}\right)^{T} \nabla^{2} g(\boldsymbol{x})\right|_{\boldsymbol{x}=\boldsymbol{x}_{0}}\left(\boldsymbol{x}-\boldsymbol{x}_{0}\right)+o\left(\left\|\boldsymbol{x}-\boldsymbol{x}_{0}\right\|^{2}\right)
\end{aligned}
$$

where $\nabla g$ and $\nabla^{2} g$ denote the gradient and the Hessian matrix of $g$, respectively. We further have

$$
\begin{aligned}
& \left.\mathbb{D}\left(P_{Z, n} \| P_{Z_{o}, n}\right)\right|_{\boldsymbol{\mu}_{n}=\mathbf{0}}=0 \\
& \left.\nabla \mathbb{D}\left(P_{Z, n} \| P_{Z_{o}, n}\right)\right|_{\boldsymbol{\mu}_{n}=\mathbf{0}}=\mathbf{0} \\
& \left.\nabla^{2} \mathbb{D}\left(P_{Z, n} \| P_{Z_{o}, n}\right)\right|_{\boldsymbol{\mu}_{n}=\mathbf{0}}=\left[\begin{array}{cc}
\frac{(\bar{q}-q)^{2}}{q \bar{q}} & -\frac{(\bar{q}-q)^{2}}{q \bar{q}} \\
-\frac{(\bar{q}-q)^{2}}{q \bar{q}} & \frac{(\bar{q}-q)^{2}}{q \bar{q}}
\end{array}\right] .
\end{aligned}
$$

Inserting (28)-(30) into (27) gives (26).

From (26), the bound (25) is fulfilled for sufficiently large $n$ if

$$
\left|a n^{\alpha}-b n^{\beta}\right| \leq k \sqrt{n} \quad \text { with } \quad k=\frac{\sqrt{2 q \bar{q}}}{\bar{q}-q} \sqrt{\delta} .
$$

Alice can thus determine achievable values of $(a, \alpha)$ if Warren expects her to send with total transmit energy $b n^{\beta}$. Alternatively, Alice can determine how much energy to invest for obfuscation to keep Warren confused when she transmits information.

A trivial but intuitive choice is $a n^{\alpha}=b n^{\beta}$. In this case, $P_{Z, n}=P_{Z_{o}, n}$ and $\mathbb{D}\left(P_{Z, n} \| P_{Z_{o}, n}\right)=0$. Moreover, if $\beta>\frac{1}{2}$ (or $\alpha>\frac{1}{2}$ ), this is the only choice for which (31) holds for all $n$. If we consider a fixed number of channel uses $n$, however, we can choose any values of $a n^{\alpha}$ and $b n^{\beta}$ satisfying (31).

For large $n$, the left-hand side (LHS) of $(31)$ is dominated by the maximum exponent $\max (\alpha, \beta)$. If $\beta \leq \frac{1}{2}$, Alice could 


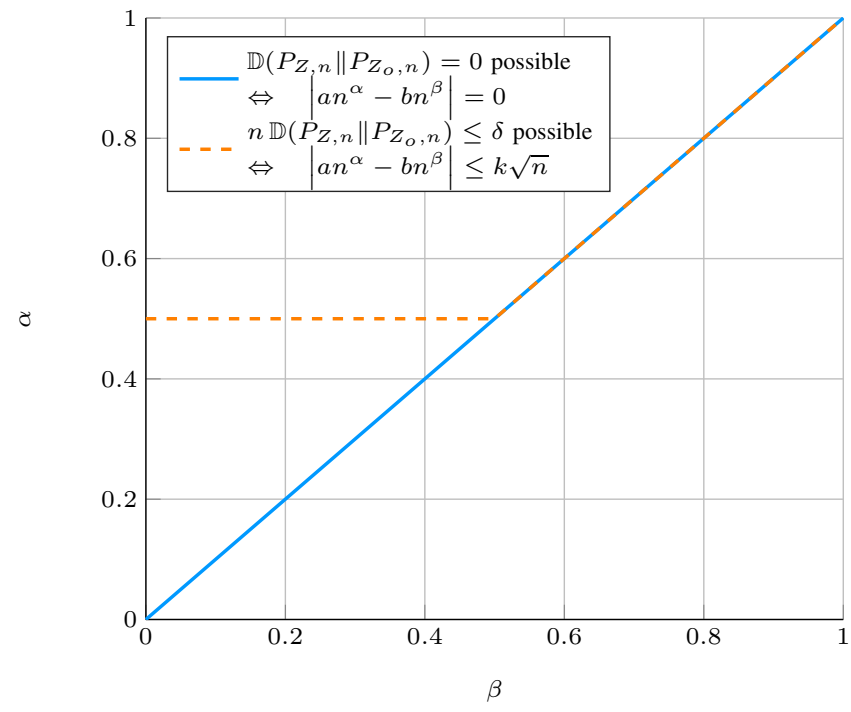

Fig. 2. Information exponent $\alpha$ vs. obfuscation exponent $\beta$.

choose $\alpha=\frac{1}{2}$ and still satisfy the stealth constraint (31). Fig. 2 summarizes the achievable information exponents $\alpha$ as a function of the obfuscation exponent $\beta$.

Consider now the covert communication scenario where $\frac{b n^{\beta}}{n}=0$. The LHS of (31) simplifies to

$$
a n^{\alpha} \leq k \sqrt{n}
$$

and allows Alice to set $\alpha=\frac{1}{2}$ and $a=k$ for any $n$. We recover the square root law for covert communication with maximum codebook scaling constant (see (21))

$$
R_{\frac{1}{2}, \max }=k(1-2 p) \log \frac{\bar{p}}{p}=\frac{\sqrt{2 q \bar{q}} \cdot(1-2 p)}{1-2 q} \sqrt{\delta} \log \frac{\bar{p}}{p} .
$$

Fig. 2 might give the impression that if one can transmit with $\alpha=\frac{1}{2}$ even if $\frac{b n^{\beta}}{n}=0$, then spending energy on obfuscation does not help in transmitting more information unless $\beta>0.5$. However, consider the case $\beta=\frac{1}{2}$. As before, Alice can choose the information exponent also to be $\alpha=\frac{1}{2}$. The LHS of (31) now reduces to $|a-b| \leq k$. This allows Alice to choose

$$
a=k+b
$$

which translates into an increased maximum square root scaling constant $R_{\frac{1}{2} \text {, max }}$ compared with (33).

\section{B. Coded Stealth}

Consider the following random coding experiment. Alice generates $M K$ codewords $X^{n}(w, v), w=1, \ldots, M, v=$ $1, \ldots, K$, where the codeword symbols are choosen i.i.d. according to $P_{X, n}$. Let $\tilde{\mathcal{C}}=\left\{X^{n}(1,1), \ldots, X^{n}(M, K)\right\}$ be the random codebook and let all codewords be equiprobable. Further, Alice and Bob share a secret key $\tilde{v}$ drawn uniformly from $\{1, \ldots, K\}$. Let $\tilde{\mathcal{C}}_{\tilde{v}}=\left\{X^{n}(1, \tilde{v}), \ldots, X^{n}(M, \tilde{v})\right\}$ be the corresponding subcodebook.

Alice: Given a message $w$ and the key $\tilde{v}$, Alice transmits the codeword $x^{n}(w, \tilde{v})$ from the subcodebook $\tilde{\mathcal{C}}_{\tilde{v}}$.
Bob: Bob observes the output $y^{n}$ of his $\operatorname{BSC}(p)$ from Alice. As he knows that Alice used the subcodebook $\mathcal{C}_{\tilde{v}}$, he finds his ML estimate as

$$
\hat{w}=\underset{w^{\prime} \in\{1, \ldots, M\}}{\operatorname{argmax}} P_{Y^{n} \mid X^{n}}\left(y^{n} \mid x^{n}\left(w^{\prime}, \tilde{v}\right)\right) .
$$

Warren: Warren observes the output $z^{n}$ of his $\operatorname{BSC}(q)$. To detect whether Alice was transmitting information to Bob or not, he runs a binary hypothesis test. As he does not know the secret key $\tilde{v}$, he must test against the entire $\operatorname{codebook} \tilde{\mathcal{C}}$.

Reliability: Both Alice and Bob know that subcodebook $\tilde{\mathcal{C}}_{\tilde{v}}$ was used. As the $n M$ symbols of $\tilde{\mathcal{C}}_{\tilde{v}}$ are sampled from $P_{X, n}$, we can apply the modified error exponents from Sec. III-B. According to (21), the probability of decoding error can be made small as long as

$$
R_{\alpha}<a(1-2 p) \log \frac{\bar{p}}{p} .
$$

Stealth: Warren observes $Z^{n}$ with the distribution

$$
P_{Z^{n} \mid \tilde{\mathcal{C}}}\left(z^{n} \mid \tilde{\mathcal{C}}\right)=\sum_{w=1}^{M K} \frac{1}{M K} P_{Z \mid X}^{n}\left(z^{n} \mid X^{n}(w, v)\right) .
$$

To keep Warren confused, Alice must therefore ensure that

$$
\mathbb{E}\left[\mathbb{D}\left(P_{Z^{n} \mid \tilde{\mathcal{C}}} \| P_{Z_{o}}^{n}\right)\right] \leq \theta
$$

for a small constant $\theta>0$.

Let $R_{\alpha}^{M K}=(\log M+\log K) / n^{\alpha}$ be the scaling constant of the code $\tilde{\mathcal{C}}$. The stealth constraint $(38)$ is satisfied by choosing

$$
R_{\alpha}^{M K}>a(1-2 q) \log \frac{\bar{q}}{q}
$$

where $a$ satisfies the uncoded stealth constraint (31) for an appropriately small constant $\delta>0$.

Proof: We split $\mathbb{E}\left[\mathbb{D}\left(P_{Z^{n} \mid \tilde{\mathcal{C}}} \| P_{Z_{o}}^{n}\right)\right]$ into three parts:

$$
\begin{aligned}
& \mathbb{E}\left[\mathbb{D}\left(P_{Z^{n} \mid \tilde{\mathcal{C}}} \| P_{Z_{o}}^{n}\right)\right]=\underbrace{\mathbb{E}\left[\mathbb{D}\left(P_{Z^{n} \mid \tilde{\mathcal{C}}} \| P_{Z}^{n}\right)\right]}_{(a)}+\underbrace{\mathbb{D}\left(P_{Z}^{n} \| P_{Z_{o}}^{n}\right)}_{(b)} \\
& +\underbrace{\mathbb{E}\left[\left(\sum_{z_{n}} P_{Z^{n} \mid \tilde{\mathcal{C}}}\left(z^{n} \mid \tilde{\mathcal{C}}\right)-P_{Z}^{n}\left(z^{n}\right)\right) \log \frac{\left.P_{Z}^{n}\left(z^{n}\right)\right]}{\left.P_{Z_{o}}^{n}\left(z^{n}\right)\right]}\right.}_{(c)} .
\end{aligned}
$$

Bounding $(a)$ is a standard resolvability problem [10], [3]. We follow the proof technique from [11, Lemma 2], [12, Sec. IIIB], [13, Sec. 5.2.2, Lemma 5.3] that develops resolvability exponents, and we adapt it to our VP transmission setting. Consider $-\frac{1}{2} \leq \rho \leq 0$. Since the modified error exponent $\hat{E}_{0}^{\alpha}$ as in (14) and (18) becomes negative for these values of $\rho$, we define the modified resolvability exponents as

$$
\begin{aligned}
& \hat{E}_{r}^{\alpha}\left(\rho, P_{X, n}\right)=-\hat{E}_{0}^{\alpha}\left(\rho, P_{X, n}\right) \\
& \hat{E}_{R}^{\alpha}\left(R_{\alpha}, P_{X, n}\right)=\inf _{-\frac{1}{2} \leq \rho \leq 0}\left(\hat{E}_{0}^{\alpha}\left(\rho, P_{X, n}\right)+\rho R_{\alpha}^{M K}\right) .
\end{aligned}
$$


The analysis in Sec. II-B also holds for (41) and (42), and we obtain

$$
\begin{cases}\hat{E}_{R}^{\alpha}\left(R_{\alpha}^{M K}, P_{X, n}\right)<0 & \text { if } R_{\alpha}^{M K}>a(1-2 q) \log \frac{\bar{q}}{q} \\ \hat{E}_{R}^{\alpha}\left(R_{\alpha}^{M K}, P_{X, n}\right)=0 & \text { if } R_{\alpha}^{M K} \leq a(1-2 q) \log \frac{\bar{q}}{q}\end{cases}
$$

Next, following [11, Sec. III], the average divergence $\mathbb{E}\left[\mathbb{D}\left(P_{Z^{n} \mid \tilde{\mathcal{C}}} \| P_{Z}^{n}\right)\right]$ is the mutual information $I\left(\tilde{\mathcal{C}} ; Z^{n}\right)$ of the codebook $\tilde{\mathcal{C}}$ and the channel output $Z^{n}$. We therefore define

$$
\hat{E}_{r}^{\alpha, n}\left(\rho, P_{X^{n}}\right)=\log \sum_{z^{n}}\left\{\mathbb{E}\left[P_{Z^{n} \mid \tilde{\mathcal{C}}}\left(z^{n} \mid \tilde{\mathcal{C}}\right)\right]^{\frac{1}{1+\rho}}\right\}^{1+\rho}
$$

which has the following properties [11, Lemma 2]:

$$
\begin{aligned}
& \hat{E}_{r}^{\alpha, n}\left(0, P_{X^{n}}\right)=\hat{E}_{r}^{\alpha}\left(0, P_{X, n}\right)=0 \\
& \left.\frac{\partial \hat{E}_{r}^{\alpha, n}\left(\rho, P_{X, n}\right)}{\partial \rho}\right|_{\rho=0}=-\mathbb{E}\left[\mathbb{D}\left(P_{Z^{n} \mid \tilde{\mathcal{C}}} \| P_{Z}^{n}\right)\right] \\
& \left.\frac{\partial^{2} \hat{E}_{r}^{\alpha, n}\left(\rho, P_{X, n}\right)}{\partial \rho^{2}}\right|_{\rho=0} \geq 0 .
\end{aligned}
$$

A slight modification of the proof of [12, Lemma 2], [13 Lemma 5.3] where we replace the codebook size by $\log |\tilde{\mathcal{C}}|=$ $n^{\alpha} R_{\alpha}^{M K}$ in [12, Eq. (46)] yields

$$
\begin{aligned}
\hat{E}_{r}^{\alpha, n}\left(0, P_{X^{n}}\right) & \leq \log \left(1+e^{n^{\alpha}} \hat{E}_{R}^{\alpha}\left(R_{\alpha}^{M K}, P_{X, n}\right)\right. \\
& \leq e^{n^{\alpha} \hat{E}_{R}^{\alpha}\left(R_{\alpha}^{M K}, P_{X, n}\right)} .
\end{aligned}
$$

By combining 45-47), we obtain

$$
\rho \cdot\left(-\mathbb{E}\left[\mathbb{D}\left(P_{Z^{n} \mid \tilde{\mathcal{C}}} \| P_{Z}^{n}\right)\right]\right) \leq \hat{E}_{r}^{\alpha, n}\left(\rho, P_{X^{n}}\right)
$$

for $-\frac{1}{2} \leq \rho \leq 0$, and thus

$$
\begin{aligned}
\mathbb{E}\left[\mathbb{D}\left(P_{Z^{n} \mid \tilde{\mathcal{C}}} \| P_{Z}^{n}\right)\right] & \leq \frac{\hat{E}_{r}^{\alpha, n}\left(0, P_{X^{n}}\right)}{-\rho} \\
& \leq \frac{e^{n^{\alpha} \hat{E}_{R}^{\alpha}\left(R_{\alpha}^{M K}, P_{X, n}\right)}}{-\rho}
\end{aligned}
$$

where we used (48) in the last step. By (50) and (43), we see that the term $(a)$ in (40) goes to zero for $n \rightarrow \infty$ if $R_{\alpha}^{M K}>a(1-2 q) \log \frac{\bar{q}}{q}$.

To bound the term $(b)$ in (40) we note that $\mathbb{D}\left(P_{Z}^{n} \| P_{Z_{o}}^{n}\right)=$ $n \mathbb{D}\left(P_{Z, n} \| P_{Z_{o}, n}\right)$. We can therefore reuse our results for uncoded stealth and must only ensure that we satisfy (31) for a small enough constant $\delta, 0<\delta<\theta$.

Finally, we rewrite term (c) in (40) as follows:

$$
\begin{aligned}
& \left|\mathbb{E}\left[\left(\sum_{z_{n}} P_{Z^{n} \mid \tilde{\mathcal{C}}}\left(z^{n} \mid \tilde{\mathcal{C}}\right)-P_{Z}^{n}\left(z^{n}\right)\right) \log \frac{P_{Z}^{n}\left(z^{n}\right)}{P_{Z_{o}}^{n}\left(z^{n}\right)}\right]\right| \\
& \leq 2 n \mathbb{E}\left[\mathbb{V}\left(P_{Z^{n} \mid \tilde{\mathcal{C}}} \| P_{Z}^{n}\right)\right] \log \frac{1}{\nu_{d}} \\
& \leq 2 n \sqrt{\frac{1}{2} \mathbb{E}\left[\mathbb{D}\left(P_{Z^{n} \mid \tilde{\mathcal{C}}} \| P_{Z}^{n}\right)\right]} \log \frac{1}{\nu_{d}} \\
& \leq \sqrt{2} n \cdot \frac{e^{\frac{1}{2} n^{\alpha} \hat{E}_{R}^{\alpha}\left(R_{\alpha}^{M K}, P_{X, n}\right)}}{\sqrt{-\rho}} \log \frac{1}{\nu_{d}}
\end{aligned}
$$

where we used (2) and $\nu_{d}=\min _{z} P_{Z_{o}, n}(z)$ in (51), where (52) follows by Pinsker's inequality (3) and Jensen's inequality, and where we reused the bound (50) in (53). Again, the RHS of (53) goes to zero as $n \rightarrow \infty$ if $R_{\alpha}^{M K}>a(1-2 q) \log \frac{\bar{q}}{q}$.

Summarizing (36) and (39), for small positive $\xi$ we can bound

$$
\begin{aligned}
& \log M \leq n^{\alpha}(1-\xi) a(1-2 p) \log \frac{\bar{p}}{p} \\
& \log K \geq \\
& n^{\alpha}\left[(1+\xi) a(1-2 q) \log \frac{\bar{q}}{q}-(1-\xi) a(1-2 p) \log \frac{\bar{p}}{p}\right]^{+}
\end{aligned}
$$

where $[x]^{+}=\max (x, 0)$ and where $\alpha, a$ satisfy (31) for specified $\beta, b$.

\section{Discussion}

We compare our results to bounds derived in [2], [3], [6]. The work in [2] considers covert communication where Warren and Bob both observe channel outputs from a $\operatorname{BSC}(q)$. Moreover, the channel outputs are i.i.d. also when Alice transmits information to Bob, which is equivalent to our uncoded stealth scenario from Sec. IV-A with $\alpha=\frac{1}{2}$ and $\frac{b n^{\beta}}{n}=0$. The maximum scaling constant (33) reduces to $R_{\frac{1}{2} \text {,max }}=\sqrt{2 q \bar{q}} \sqrt{\delta} \log \frac{\bar{q}}{q}$, which is the same value one would obtain from [2, Thm. 2] for BSCs.

Similarly, we compare our coded results (54) and (55) to the bounds in Corollary 2 of Thm. 2 in [3] for the covert communication scenario. From (31) we know that Alice can choose at most $\alpha=\frac{1}{2}$ and $a=k$. Further, $k$ can be alternatively expressed as

$$
k=\sqrt{\frac{2}{\chi_{2}\left(P_{Z \mid X}(\cdot \mid 1) \| P_{Z \mid X}(\cdot \mid 0)\right)}} \cdot \sqrt{\delta}
$$

where $\delta<\mathbb{E}\left[\mathbb{D}\left(P_{Z^{n} \mid \tilde{\mathcal{C}}} \| P_{Z_{o}}^{n}\right)\right]$. Our bounds then match the ones from [3, Corollary 2] when evaluated for BSCs.

Our results also apply to covert communication without a secret key by choosing $\log K=0$. The codebook scaling constant $R_{\alpha}$ is then upper and lower bounded by the RHSs of (36) and (39), respectively. These are exactly the same bounds reported in [6, Thm. 1] for BSCs and variational distance as the stealth measure, where the authors assumed that Bob's channel from Alice must be better than Warren's. Note that without a secret key, one must have $p<q$ to satisfy the bounds. Moreover, (55) implies that the key size can be zero if $p<q$.

We conclude with two remarks. First, due to space constraints we presented only the achievability proof and left the converse proof for a future document. Second, we have studied BSCs only; extensions to general DMCs will be treated in a future document.

\section{ACKNOWLEDGMENT}

This work was supported by the German Research Foundation (DFG) under Grant KR 3517/9-1. 


\section{REFERENCES}

[1] B. A. Bash, D. Goeckel, and D. Towsley, "Limits of reliable communication with low probability of detection on AWGN channels," IEEE J. Sel. Areas Commun., vol. 31, no. 9, pp. 1921-1930, Sep. 2013.

[2] L. Wang, G. W. Wornell, and L. Zheng, "Fundamental limits of communication with low probability of detection," IEEE Trans. Inf. Theory, vol. 62, no. 6, pp. 3493-3503, Jun. 2016.

[3] M. R. Bloch, "Covert communication over noisy channels: A resolvability perspective," IEEE Trans. Inf. Theory, vol. 62, no. 5, pp. 2334-2354, May 2016.

[4] M. Tahmasbi and M. R. Bloch, "First- and second-order asymptotics in covert communication," IEEE Trans. Inf. Theory, vol. 65, no. 4, pp. 2190-2212, Apr. 2019.

[5] P. H. Che, M. Bakshi, and S. Jaggi, "Reliable deniable communication: Hiding messages in noise," in Proc. IEEE Int. Symp. Inf. Theory (ISIT), Istanbul, Turkey, Jul. 2013, pp. 2945-2949.

[6] P. H. Che, M. Bakshi, C. Chan, and S. Jaggi, "Reliable, deniable and hidable communication," in Proc. Inf. Theory and Applicat. Workshop (ITA), San Diego, CA, USA, Feb. 2014, pp. 1-10.
[7] J. Hou and G. Kramer, "Effective secrecy: Reliability, confusion and stealth," in Proc. IEEE Int. Symp. Inf. Theory (ISIT), Honolulu, HI, USA, Jun. 2014, pp. 601-605.

[8] J. Hou, G. Kramer, and M. Bloch, "Effective secrecy: reliability, confusion and stealth," in Information Theoretic Security and Privacy of Information Systems, H. Boche, A. Khisti, H. V. Poor, and R. F. Schaefer, Eds. Cambridge Univ. Press, 2017, pp. 3-20.

[9] R. G. Gallager, Information Theory and Reliable Communication. New York, NY, USA: John Wiley \& Sons, Inc., 1968.

[10] T. S. Han and S. Verdu, "Approximation theory of output statistics," IEEE Trans. Inf. Theory, vol. 39, no. 3, pp. 752-772, May 1993

[11] M. Hayashi, "General nonasymptotic and asymptotic formulas in channel resolvability and identification capacity and their application to the wiretap channel," IEEE Trans. Inf. Theory, vol. 52, no. 4, pp. 15621575, Apr. 2006.

[12] J. Hou and G. Kramer, "Informational divergence approximations to product distributions," in Proc. Canadian Workshop Inf. Theory (CWIT), Toronto, ON, Canada, Jun. 2013, pp. 76-81.

[13] J. Hou, "Coding for relay networks and effective secrecy for wire-tap channels," Ph.D. dissertation, Technical University of Munich, 2014. 